DOI: $10.17516 / 1997-1370-0785$

УДК 504.03

\title{
Toward the Sustainable Development of Russian Regions: A Comprehensive Review of Empirical Research
}

\author{
Yulia I. Pyzheva*a, Evgeniya V. Zander ${ }^{\text {a }}$ \\ and Anton I. Pyzheva, b \\ ${ }^{a}$ Siberian Federal University \\ Krasnoyarsk, Russian Federation \\ ${ }^{b}$ Institute of Economics and Industrial Engineerin, SB RAS \\ Novosibirsk, Russian Federation
}

Received 29.05.2021, received in revised form 21.06.2021, accepted 12.07.2021

\begin{abstract}
The paper provides a comprehensive review of empirical research on the sustainability of development of Russian regions. Methods of bibliometric analysis are used to achieve the set task. Samples of publications for substantive analysis are constructed using the leading academic databases: Scientific electronic library eLIBRARY.ru and its project Russian Science Citation Index (RSCI), Scopus, Web of Science. The results of the study show that despite the increasing public and political attention to the problems of sustainability, ecologization of the economy, improving the well-being of the population, there are few high-quality scientific studies that make a significant contribution to the analysis of sustainability of the Russian regions. Most of the research is of a staged or descriptive nature, and there are very few quantitative studies that are in such high demand on the modern international scientific market. For example, assessments of sustainability levels have covered only a small part of Russian regions over a very short period not exceeding five years on average, and using a very narrow range of tools. This is clearly insufficient for use as alternatives to traditional macroeconomic measures of well-being of territories, which significantly narrows the demand for these results in practice. The shortage of publications on the topic of sustainability of Russian regions can be perceived as a direction for the development of relevant research, opening new directions and even creating new teams.
\end{abstract}

Keywords: sustainable development, economy, socio-economic development, Russian regions, research review, bibliometric analysis, scientific productivity.

The reported study was funded by RFBR, project number 20-110-50580.

Research area: economics.

\footnotetext{
(C) Siberian Federal University. All rights reserved

* Corresponding author E-mail address: ystartseva@sfu-kras.ru

ORCID: 0000-0003-0973-5073 (Pyzheva); 0000-0002-2135-5084 (Zander); 0000-0001-7909-3227 (Pyzhev)
} 
Citation: Pyzheva Yu.I., Zander E. V., Pyzhev A. I. (2021). Toward the sustainable development of russian regions: a comprehensive review of empirical research. J. Sib. Fed. Univ. Humanit. Soc. Sci., 14(7), 1063-1079. DOI: 10.17516/1997-1370-0785.

\title{
На пути к устойчивому развитию российских регионов: комплексный обзор эмпирических исследований
}

\author{
Ю.И. Пыжева ${ }^{a}$ Е.В. Зандера , А.И. Пыжев ${ }^{a, 6}$ \\ ${ }^{a}$ Сибирский федеральный университет \\ Российская Федерачия, Красноярск \\ ${ }^{6}$ Институт экономики и организации \\ промышленного производства СО РАН \\ Российская Федерация, Новосибирск
}

\begin{abstract}
Аннотация. В статье дается комплексный обзор эмпирических исследований устойчивости развития российских регионов. Для достижения поставленной задачи использованы современные методы библиометрического анализа. Выборки публикаций для содержательного анализа построены с использованием ведущих академических баз данных: Научной электронной библиотеки eLIBRARY.ru (HЭБ) и eе проекта Российского индекса научного цитирования (РИНЦ), Scopus, Web of Science. Результаты исследования показывают, что несмотря на усиливающееся общественнополитическое внимание к проблемам устойчивости развития, экологизации экономики, повышения благополучия населения, качественных научных исследований, вносящих существенный вклад в анализ устойчивости развития российских регионов, немного. Большая часть исследований носит постановочный или описательный характер, а количественных исследований, столь востребованных на современном международном научном рынке, очень мало. Например, оценками уровней устойчивости развития покрыта лишь малая часть российских регионов, причем за очень небольшой период, не превышающий в среднем пяти лет и с применением очень узкого круга инструментов. Этого явно недостаточно для использования в качестве альтернатив традиционным макроэкономическим мерам благополучия развития территорий, что существенно сужает востребованность данных результатов практикой. Дефицит публикаций по тематике устойчивости российских регионов можно воспринимать как направление для развития соответствующих исследований, открытия новых направлений и даже создания новых коллективов.
\end{abstract}

Ключевые слова: устойчивое развитие, экономика, социо-эколо-экономическое развитие, российские регионы, обзор исследований, библиометрический анализ, научная продуктивность.

Исследование выполнено при финансовой поддержке РФФИ в рамках научного проекта № 20-110-50580.

Научная специальность: 08.00.05 - экономика и управление народным хозяйством. 


\section{Введение}

В последние десятилетия в обществе формируется новая повестка дня, одно из ключевых мест в которой занимают вопросы влияния экологических, экономических и социальных факторов на развитие стран, регионов и муниципалитетов. По мере роста совокупного богатства населения планеты всё более актуальными будут вопросы, связанные с повышением качества и продолжительности жизни. По данным Всемирного Банка, с 1990 года ВВП стран низкого и среднего дохода ${ }^{1}$ увеличился более чем вдвое, а продолжительности жизни - с 63,2 до 68,6 лет (UNDP, 2017). Несмотря на столь существенный прогресс, на глобальном уровне сохраняют остроту проблемы голода, нищеты, ограниченного доступа к элементарным бытовым услугам и пр. Таким образом, сохраняется проблема существенного неравенства доходов и богатства населения, которая обостряет общественный запрос на справедливое распределение глобальных ресурсов. Важно подчеркнуть, что в последнее время к сугубо социальной природе такого запроса всё чаще присоединяются требования, связанные с качеством окружающей среды.

Сформулированная в конце 1980-х годов идея устойчивости развития (англ. sustainable development), то есть такого развития, которое позволяет удовлетворить потребности нынешнего поколения без существенного ущерба для благополучия будущих поколений, прочно вошла в повседневную жизнь общества в развитых странах мира. Из призывов к решению достаточно абстрактных глобальных проблем устойчивость превратилась для ведущих стран мира в действующий инструмент формирования новых потребительских привычек населения, связанных, прежде всего, с идеями «зеленого мышления», ответственного потребления и пр. (Tambovceva et al., 2018; Ali et al., 2020). Различные метрики устой-

\footnotetext{
1 Например, Россия по данной классификации находится в категории стран с доходом выше среднего, то есть страной, для которой валовой национальный доход на душу населения находится в диапазоне 4 046-12 535 долл. США (фактическое значение в 2020 году составляет 11260 долл. США).
}

чивости, например объем выбросов углекислого газа от авиационного транспорта, теперь учитывается при расчете тарифов на авиаперевозки. Между тем подобные инициативы хоть и лежат в русле концепции устойчивости, но несколько отходят от ее первоначальной сути: обеспечения сбалансированного роста благосостояния населения при обеспечении гармоничных социальных и экологических характеристик качества жизни.

Отдельно отметим, что здесь и далее рассматривается устойчивость в применении к экономическим проблемам, поскольку довольно часто данный термин встречается, например, в экологической литературе и в целом тематически пересекается с исследованиями «экономической» устойчивости (Malik, 2012). Тем не менее в целях данного исследования используется именно вышеописанный подход, сконцентрированный исключительно на экономической академической литературе с соответствующими данной тематике научными результатами.

В России еще десять лет назад эти идеи были даже не знакомы большей части населения, однако в последние годы запрос общества на изменение экономической политики с учетом экологических и социальных ограничений постепенно становится всё более выраженным. Очевидно, что эта тенденция будет сохраняться по мере роста благосостояния населения и смены поколений: молодые люди, особенно проживающие в крупных городах, предъявляют гораздо больший спрос на качественную окружающую среду и социальные услуги. Всё это подчеркивает актуальность социально-экономических исследований в области устойчивости развития.

Огромное физико-географическое пространство России предопределяет высокую степень неравномерности развития отдельных территорий, поэтому исследования устойчивости особенно актуальны именно на региональном уровне (Kryukov, Kolomak, 2021; Kolomak, 2019; Demyanenko et al., 2012). За три постсоветских десятилетия в российской литературе накопи- 
лось довольно много исследований устойчивости развития регионов, в том числе дающих сплошные региональные динамические оценки разрывов между устойчивостью и экономическим ростом (Bobylev et al., 2012; Glazyrina et al., 2005; Ryumina, Anikina, 2009). С учетом того, что сам по себе термин «устойчивость» стал интерпретироваться в рамках научных школ или небольших исследовательских групп самым различным образом, а комплексных работ, которые бы давали себе цель обобщить все эти результаты, практически нет, такая задача представляется перспективной и важной для дальнейшего развития данного научного направления.

Интенсивное внедрение компьютерной техники и рост количества публикаций в последние десятилетия предопределили интенсивное развитие библиометрических исследований в различных областях наук (Quental, Lourenço, 2012; Eker et al., 2019; Hallinger, 2021). Интерес к подобного рода исследованиям наблюдается и в России, однако их количество невелико, а охват по-прежнему недостаточен (Mkrtchyan et al., 2015; Demyanenko et al., 2012; Muravyev, 2011; Pyzhev, 2021). Между тем современный поток публикаций по любой самой узкой теме настолько велик, что необходимы регулярные метааналитические исследования срезов текущих работ, с помощью которых можно было бы не только сориентировать в новой теме тех, кто только начал ей заниматься, но и сформировать представление о действительной степени разработанности проблемы и тех вопросов, над разрешением которых необходимо работать в будущем.

Настоящее исследование предлагает систематизировать корпус российской литературы, посвященной эмпирическим исследованиям устойчивости развития регионов страны. Для достижения поставленной задачи будут использованы современные методы библиометрического анализа. Будут рассмотрены как российские, так и международные исследования, индексированные ведущими базами данных: Научной электронной библиотекой eLIBRARY. ru (НЭБ) и ее проекта Российского индекса научного цитирования (РИНЦ), Scopus, Web of Science.

Базовая гипотеза исследования заключается в том, что несмотря на формально большое количество эмпирических исследований, качественных работ, вносящих существенный научный вклад в анализ устойчивости развития российских регионов, немного. Также остается еще целый ряд инструментов, широко известных в мировой науке, но так и не нашедших применения в России для исследований по данной тематики. Кроме того, очень мало оригинальных разработок, которые могли бы быть «экспортированы», то есть применены в других странах для соответствующих межстрановых и межрегиональных сопоставлений.

Мы убеждены, что решение данной задачи необходимо для того, чтобы оценить полноту и качество эмпирических результатов, полученных в данной области, а также для выделения направлений для проведения новых работ, способных внести существенный вклад в понимание того, как развиваются российские территории. Качественная аналитическая информация по этой теме необходима для разработки и реализации эффективных стратегий социо-эколого-экономического развития страны.

\section{Методы}

Изолированность российского научного рынка от мирового предопределяет необходимость использования для комплексного обзора литературы как общепризнанных международных баз данных научных публикаций, так и российских аналогов, поскольку большая доля качественных научных статей пишется и издается только на русском языке (Muravyev, 2011). Данная ситуация меняется в последние годы в связи со стимулированием публикаций в рамках грантовых программ и базовой отчетности академических институтов и университетов, однако говорить о полном уходе от русскоязычных публикаций преждевременно. 
Аналитические возможности используемых баз данных научных публикаций (Web of Science, Scopus, РИНЦ) различаются, но в целом позволяют оценивать сопоставимые показатели без существенных ограничений или искажений результатов. В рамках представленного исследования предлагается использовать базовые методы библиометрического анализа, основанные, прежде всего, на встроенных инструментах интернет-порталов соответствующих баз данных. Как показал опыт предыдущих исследований, применение более развитых методов кластеризации и визуализации библиометрических исследований для выборок по российским экономическим исследованиям практически бессмысленно ввиду их малочисленности (Pyzhev, 2021). Расчет некоторых метрик по международным базам данных будет проводиться с помощью пакета bibliometrix (Aria, Cuccurullo, 2017) в программной среде обработки статистических данных R (R Core Team, 2020).

Результаты анализа основаны на выборках публикаций, подготовленных по соответствующим запросам и с выделением подходящих фильтров (табл. 1). Отдельно отметим, что попытка замены термина «регион» на термин «субъект» (в значении: «субъект Российской Федерации») в поисковом запросе не привела к получению дополнительных полезных для настоящей работы результатов, поскольку выборки с этим термином во всех случаях (250 результатов) указывают на «хозяйствующие субъекты» или «субъекты малого предпринимательства». Аналогично термин «территория» вместо «региона» приводит к обширной выборке публикаций по устойчивости сельскохозяйственных территорий.

В выборки по русскоязычным источникам включались только статьи и книги (монографии), поскольку именно такие виды публикаций содержат первичные результаты исследований. Материалы докладов и диссертаций здесь представляются вторичным источником, хотя, разумеется, в некоторых исключительных случаях определенные результаты содержатся только в них, но не раскрываются в статьях соответствующих авторов. Тем не менее исключение данных видов публикаций из рассмотрения представляется разумным компромиссом между трудоемкостью обработки первичных данных и пользой для дальнейшего анализа. В то же время было решено не накладывать аналогичное ограничение на выборки из международных баз ввиду 1) малого количества публикаций, 2) того факта, что в таких базах не индексируются короткие тезисы (объемом до 4000 знаков), которые распространены

Таблица 1. Описания поисковых запросов и фильтров, использованных для формирования выборок публикаций по тематике устойчивости развития регионов России

Table 1. Descriptions of search queries and filters used to generate a sample of publications on the topic of sustainability of Russia's regions

\begin{tabular}{|c|c|c|}
\hline База данных & Запрос & Фильтры \\
\hline РИНЦ & $\begin{array}{l}\text { устойчивое развитие } \\
\text { региона, устойчивость } \\
\text { развития региона }\end{array}$ & $\begin{array}{l}\text { Статьи в журналах, книги (монографии). Поиск } \\
\text { с учетом морфологии, по названию публикации } \\
\text { и ключевым словам. Публикации до } 2020 \text { года } \\
\text { включительно }\end{array}$ \\
\hline Scopus & 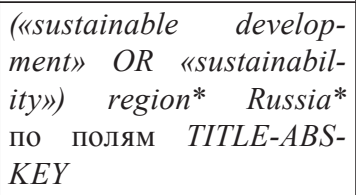 & $\begin{array}{l}\text { Тематики: Economics, Econometrics and Finance; } \\
\text { Social Sciences; Multidisciplinary. Публикации } \\
\text { до } 2020 \text { года включительно }\end{array}$ \\
\hline Web of Science & $\begin{array}{l}\text { («sustainable develop- } \\
\text { ment» OR «Sustainabili- } \\
\text { ty») region* Russia* }\end{array}$ & $\begin{array}{l}\text { Тематики: Economics; Area Studies; Multidisci- } \\
\text { plinary. Публикации до } 2020 \text { года включительно }\end{array}$ \\
\hline
\end{tabular}


в РИНЦ, и учебно-методическая литератуpa, по определению не содержащая новых научных результатов.

Отсутствие эффективных входных барьеров для недобросовестных изданий в РИНЦ объясняет низкое среднее качество публикаций, которые индексируются в этой базе данных (Pyzhev, 2021). Осознавая данную проблему, владельцы НЭБ предпринимают работу по разделению всего пула публикаций на подвыборки и исключению из индексации изданий с выявленными нарушениями публикационной этики (Grigor'eva, Glukhov, 2017). С учетом отмеченного факта было решено в дополнение к анализу по всей совокупности публикаций РИНЦ отдельно рассматривать подвыборки из данной базы: проекта RSCI Russian Science Citation Index на платформе Web of Science и «ядра РИНЦ» (подборки публикаций РИНЦ, включающей только журналы, индексируемые в Web of Science Core Collection, Scopus и RSCI).

После проведения формализованных запросов из соответствующих баз данных полученные выборки подвергались экспертному анализу, после чего из них исключались документы, не имеющие отношения к анализируемой тематике. Проведенный таким образом сплошной анализ публикаций позволяет исключить ошибки включения в выборку нерелевантных документов и вряд ли может быть заменен каким-либо альтернативным автоматизированным методом. Как отмечалось выше, в российском научном дискурсе сложилось сразу несколько взглядов на понятие «устойчивости развития», что привело к высокому разнообразию тематик работ, которые содержат данный термин в своих метаданных (заголовке, ключевых словах, аннотации). Помимо классического понимания устойчивости как состояния равновесия триады экономического развития, состоящей из экономической, экологической и социальной компонент (Hecht, 1999), в русскоязычной литературе данный термин часто применяют к описанию чисто отраслевых работ в смысле «обеспечения устойчивости развития предприятия или отрасли».
Разумеется, такой взгляд не имеет ничего общего с концепцией устойчивого развития и является примером омонимичного восприятия терминологии при переводе с другого языка. Такие публикации необходимо отделять от тех, которые относятся непосредственно к теме исследования.

В целях экономии места в статье будут приводиться только самые важные количественные характеристики и соответствующие таблицы с данными без ущерба для содержательных выводов исследования. Тот же тезис относится к многочисленным ссылкам, существенную часть которых не представляется возможным привести в статье.

Поскольку цель настоящей работы заключается в обзоре общих тенденций развития предметного поля оценки устойчивости развития российских регионов, а не только в статистической характеристике выборок публикаций, в работе далее проводятся соответствующие аналитические обобщения, основанные на работе с источниками, отобранными в ходе библиометрического анализа.

\section{Результаты}

Выполненную работу можно условно разделить на два крупных этапа: 1) формирование совокупностей библиографических записей, подходящих под требования исследования публикаций, и проведение базовых процедур библиометрического описания и 2) последующий содержательный анализ самих публикаций. Выгрузка данных из соответствующих баз проводилась по состоянию на март 2021 года. Для обеспечения сопоставимости результатов библиометрического анализа учитывались только полные годы, начиная с 1995 года. ${ }^{2}$ После выполнения сформулированных выше запросов с учетом выбранных фильтров (табл. 1) совокупности библиографических описаний публикаций были подвержены экспертному анализу на предмет исключения нерелевантных по тематике работ. Затем были вычислены общие

\footnotetext{
2 Для целей аналитических обобщений далее были использованы все доступные публикации по тематике.
} 
Yulia I. Pyzheva, Evgeniya V. Zander... Toward the Sustainable Development of Russian Regions...

Таблица 2. Описательная статистика библиометрических показателей выборки публикаций по устойчивости развития российских регионов в ведущих академических базах данных

Table 2. Descriptive bibliometric statistics of a sample of publications on sustainability in Russian regions in selected academic databases

\begin{tabular}{|l|c|c|c|}
\hline \multicolumn{1}{|c|}{ Показатель } & $\begin{array}{c}\text { РИНЦ / ядро } \\
\text { РИНЦ / RSCI }\end{array}$ & Scopus & Web of Science \\
\hline Общее число публикаций, шт. & $3598 / 233 / 121$ & 89 & 179 \\
\hline Число авторов, чел. & $5884 / 479 / 296$ & 239 & 0,43 \\
\hline $\begin{array}{l}\text { Среднее число публикаций в расчете } \\
\text { на одного автора }\end{array}$ & $0,61 / 0,47 / 0,41$ & 0,37 & 267 \\
\hline $\begin{array}{l}\text { Суммарное число цитирований } \\
\text { публкаций }\end{array}$ & $16666 / 1451 / 805$ & 510 & 3,61 \\
\hline $\begin{array}{l}\text { Среднее число цитирований на одну } \\
\text { публикацию }\end{array}$ & $4,63 / 6,39 / 6,65$ & 6,65 & 52,0 \\
\hline $\begin{array}{l}\text { Доля публикаций, процитированных } \\
\text { хотя бы раз,\% от общего числа }\end{array}$ & $54,8 / 66,5 / 66,1$ & 74,2 & 7,8 \\
\hline $\begin{array}{l}\text { Доля самоцитирований,\% от общего } \\
\text { числа статей }\end{array}$ & $30,1 / 5,1 / 2,5^{*}$ & & 8 \\
\hline Индекс Хирша & $44 / 17 / 14$ & 11 & \\
\hline
\end{tabular}

* Рассчитаны внутри выборки публикаций, то есть без учета ссылок авторов на прочие собственные публикации.

библиометрические характеристики полученных выборок (табл. 2). Выбор показателей обуславливался целями исследования, требованиями максимально возможной сопоставимости метрик и техническими возможностями соответствующих баз данных. Некоторые показатели по БД Scopus и Web of Science (например, различные метрики цитируемости) вычислены с помощью пакета bibliometrix на основе выборок, выгруженных напрямую из баз.

РИНЦ. Как и ожидалось, количественные и качественные характеристики выборки из базы данных РИНЦ существенно отличаются от остальных рассмотренных совокупностей публикаций. Прежде всего, таких публикаций на порядок больше, но существенная их часть представляет собой обзоры, общепостановочные рассуждения о необходимости реализации политики устойчивости в отношении регионов и т. п. Во многом это связано с тем, что несмотря на исключение из рассмотрения тезисов, материалов докладов конференций и диссертаций существенная часть публикаций по РИНЦ представлена студенческими и аспирантскими работами, которые в современном пуле российских научных публикаций крайне редко являются результатами действительно оригинальных и самостоятельных исследований. Кроме того, часть публикаций, которые классифицируются как «статьи», на деле представляют собой тезисы докладов очень небольшого объема и неизвестного качества, поскольку сама по себе редакционная политика многих коммерческих изданий, публикующих подобные работы, достаточно прозрачно указывает на отсутствие какого бы то ни было отбора и рецензирования рукописей. Официально такие издатели заявляют свои издания как «рецензируемые», поскольку этого требуют не только базы данных, но и, например, Высшая аттестационная комиссия Российской Федерации при включении в свой Перечень изданий, в которых должны быть опубликованы результаты диссертационных исследований. В результате условно «качественные» статьи, прошедшие соответствующий процесс академического рецензирования, классификационно смешиваются в общем потоке с материалами, научная ценность которых не подтверждается ничем. 
Описанные факты вынуждают владельцев РИНЦ предпринимать попытки по исключению недобросовестных изданий из индексирования (Grigor'eva, Glukhov, $2017)^{3}$, однако поток таких публикаций как минимум не иссякает. Таким образом, к настоящему времени в России так и не сложилась практика надежного разделения публикаций по уровням качества даже на уровне отделения фактически тезисов (Conference Paper) от полноформатных статей с оригинальными результатами (Original Paper), как это делается в международных базах данных.

Куда больший интерес представляют собой публикации, входящие в суженные подборки: ядро РИНЦ и RSCI. Данные проекты появились в результате попыток осуществления кластеризации публикаций по научному уровню. Совместно c Web of Science в рамках деятельности НЭБ была создана т. н. «русская полка WoS», или Russian Science Citation Index (RSCI), куда вошли журналы, отобранные в результате экспертного опроса. В свою очередь, ядро РИНЦ технически добавляет публикации российских авторов в Web of Science и Scopus к публикациям в изданиях RSCI. Поскольку данные выборки пересекаются, их количественные характеристики схожи с точностью до включения в рассмотрение данных из БД Scopus и Web of Science. Ожидаемым результатом анализа таких публикаций является тот факт, что среди данных двух подвыборок существенно больше публикаций, содержащих действительно оригинальные результаты исследований. Впоследствии будут более детально проанализированы именно эти работы.

Scopus u Web of Science. Количество публикаций в данных базах невелико: в обоих случаях оно не превышает и 90 единиц за период 25 лет. Специфика включения издания в базы данных подразумевает определенное пересечение публикаций, поэтому

3 В целях соблюдения этических принципов здесь намеренно не цитируются работы, которые являются примерами недобросовестных подходов как отдельных издателей, так и авторов, пользующихся такими возможностями. общее количество публикаций едва превышает 100 единиц. Схожесть количественных характеристик выборок, полученных по БД Scopus и Web of Science, косвенно подтверждает релевантность выбранного подхода. Общие показатели цитируемости более чем скромны и свидетельствуют о достаточно низкой востребованности данных работ. Обращают на себя внимание низкие показатели самоцитируемости, что может указывать на высокую долю единичных исследований, не связанных в единый цикл работ автора или научного коллектива.

Распределение журнальных публикаций по изданиям устроено достаточно тривиально. B Web of Science лидируют издаваемая Институтом экономики Уральского отделения РАН «Экономика региона» $(\mathrm{N}=23)$ и «Экономические и социальные изменения: факты, тенденции, прогноз» ${ }^{4}$ Вологодского научного центра РАН $(\mathrm{N}=$ 10). B Scopus лидер снова «Экономика peгиона», но на втором месте - «European Research Studies Journal» $(\mathrm{N}=8)$, издаваемый греческим Университетом Пирея и исключенный из БД Scopus по неизвестным причинам в 2018 году. Прочие издания опубликовали не более 5 работ по тематике каждое. Такое распределение неудивительно: в России традиционно мало научных журналов, которые могут входить в международные базы данных, что на фоне ограниченного опыта российских исследователей в коммуникации с международным научным сообществом создает барьеры для роста количества публикаций. В качестве примера хорошей практики здесь можно привести опыт Китая, который не жалеет средств и усилий на создание большого пула собственных научных журналов, помимо наращивания объема и качества публикаций в ведущих международных изданиях (Wang et al., 2018).

Пул анализируемых исследований практически полностью замкнут на русскоязычной аудитории как по авторству, так и по потенциальному кругу читателей.

\footnotetext{
4 Данный журнал индексируется Web of Science в рамках бесквартильной коллекции Emerging Sources Citation Index, но не в Scopus.
} 
Так, например, среди организаций, указанных в аффилиациях публикаций Бд Scopus, только 4 из 53 находятся за пределами России: Университет Джорджа Вашингтона (США), Университет Мэйдзи (Япония), Университет Клагенфурта (Австрия), Технический университет Крита (Греция). Однако авторские коллективы каждой из таких статей содержат как минимум одного исследователя с именами и фамилиями очевидно российского происхождения (Shadrina, 2015; Dubina et al., 2017; Carayannis et al., 2017). При этом только один журнал, в котором опубликованы документы из выборки, входил в первый дециль по показателю CiteScore 5 . Таким образом, можно констатировать, что исследования по тематике устойчивости российских регионов носят исключительно локализованный характер и не побуждают международное сотрудничество или отдельный интерес со стороны иностранных коллективов. В целом данный вывод согласуется с результатами предыдущих исследований по другим областям экономической науки (Muravyev, 2011; Pyzhev, 2021). Интерпретацию данного эффекта можно вести с двух точек зрения. С одной стороны, это свидетельство низкого уровня развития предметного поля в силу отсутствия интереса к серьезным экономическим исследованиям по России как на внутреннем, так и международном рынке. С другой стороны, это означает большой потенциал развития подобного рода работ с достаточно низкими «порогами входа». Второй тезис можно подтвердить на основе содержательного анализа наиболее перспективного с этой точки зрения сегмента, связанного с количественными оценками устойчивости развития, которые являются наиболее важной подтемой проблематики устойчивости в мировой литературе (Hák et al., 2018).

При первичном анализе публикаций по вопросам устойчивого развития российских регионов возникает мнение, что существенная их часть посвящена оцен-

\footnotetext{
5 Рассчитывается как отношение количества цитирований к общему количеству документов за определенный период.
}

ке индикаторов или систем индикаторов устойчивости. Однако систематизация исследований с целью выделить конкретные территории, используемые инструменты и период наблюдения приводит к выводу о том, что на самом деле работы с конкретными результатами такого рода не столь многочисленны (табл. 3).

Основная часть исследований сфокусирована на отдельных регионах или субъектах, составляющих макрорегион, как правило, федеральный округ, что обусловлено особенностями организации и функционирования отечественной системы статистических наблюдений. Бо́льшая часть российских регионов не покрывается результатами исследований устойчивости развития. Исследования на уровне муниципалитетов практически не проводятся, а на уровне локальных территорий (например, малых городских или сельских поселений) - не проводятся вовсе.

Характерной особенностью большинства анализируемых исследований является достаточно скромный по длительности период наблюдений: в среднем по исследованиям, систематизированным в таблице 3 , период наблюдений составляет только 5 лет, причем есть исследования, оценивающие только статическую устойчивость (в течение одного года). Максимальный же период наблюдений в отдельных исследованиях не превышает 15 лет. Ограничения доступности статистики делают невозможными или затруднительными исследования устойчивости для периодов до 2000 года, поскольку во времена СССР и первые годы после его распада многие показатели, необходимые для соответствующих расчетов устойчивости, не наблюдались или слишком сильно колебались.

Разнообразие применяемого инструментария для оценки устойчивости также невелико. В целом все подходы к разработке подобного рода методов можно разделить на две категории: адаптация известных в международной практике индексов и систем индикаторов для применения на национальном или региональном уровне, a также создание собственных подходов. 
Таблица 3. Систематизация ключевых результатов исследования по оценке устойчивости развития российских регионов

Table 3. Systematization of key findings of the Russian regions' sustainability assessment study

\begin{tabular}{|c|c|c|c|c|}
\hline Авторы & $\begin{array}{c}\text { Объект } \\
\text { исследования }\end{array}$ & $\begin{array}{c}\text { Уровень } \\
\text { детализации }\end{array}$ & Индикатор устойчивости & Период \\
\hline 1 & 2 & 3 & 4 & 5 \\
\hline $\begin{array}{l}\text { Bobylev et al., } \\
\text { 2014, } 2018\end{array}$ & $\begin{array}{l}\text { Москва, Санкт- } \\
\text { Петербург, } \\
\text { Тюменская обл. }\end{array}$ & Города РФ & $\begin{array}{c}\text { Индекс устойчивости } \\
\text { городов, Система } \\
\text { индикаторов устойчивости } \\
\text { для городов }\end{array}$ & 2013 \\
\hline $\begin{array}{l}\text { Yakovenko } \\
\text { et al., } 2021\end{array}$ & $\begin{array}{c}\text { Воронежская } \\
\text { область }\end{array}$ & Муниципалитеты & Собственный индекс & 2014-2018 \\
\hline $\begin{array}{c}\text { Kovalenko et } \\
\text { al., } 2016\end{array}$ & $\begin{array}{c}\text { Республика } \\
\text { Мордовия }\end{array}$ & Муниципалитеты & Собственный индекс & $2010-2015$ \\
\hline $\begin{array}{l}\text { Maksimov et } \\
\text { al., } 2011\end{array}$ & $\begin{array}{c}\text { Нижегородская } \\
\text { область }\end{array}$ & Субъект РФ & $\begin{array}{c}\text { Система сбалансированных } \\
\text { показателей }\end{array}$ & 2000-2009 \\
\hline $\begin{array}{l}\text { Pyzheva et } \\
\text { al., } 2021\end{array}$ & $\begin{array}{c}\text { Регионы Дальнего } \\
\text { Востока }\end{array}$ & Субъекты РФ & Истинные сбережения & 2004-2018 \\
\hline $\begin{array}{l}\text { Gafurov et } \\
\text { al., } 2015\end{array}$ & Приволжский ФО & Субъекты РФ & $\begin{array}{c}\text { Анализ эффективности } \\
\text { деятельности органов } \\
\text { исполнительной власти } \\
\text { на основе частных } \\
\text { и интегральных показателей }\end{array}$ & 2011-2014 \\
\hline $\begin{array}{l}\text { Adam, Laptev, } \\
2015\end{array}$ & Томская область & Субъекты РФ & Индекс устойчивости & 2009-2012 \\
\hline Uskova, 2009 & $\begin{array}{c}\text { Вологодская } \\
\text { область }\end{array}$ & Субъекты РФ & $\begin{array}{c}\text { Авторский интегральный } \\
\text { индекс устойчивости, } \\
\text { частные индексы } \\
\text { экономической, социальной } \\
\text { и экологической } \\
\text { устойчивости }\end{array}$ & $\begin{array}{c}2000 \\
\text { и } 2006\end{array}$ \\
\hline $\begin{array}{l}\text { Bobylev et } \\
\text { al., } 2013\end{array}$ & Уральский ФО & Субъекты РФ & $\begin{array}{c}\text { Индекс скорректированных } \\
\text { чистых накоплений }\end{array}$ & 2012 \\
\hline Korobitsyn, 2018 & Уральский ФО & Субъекты РФ & $\begin{array}{c}\text { Коэффициенты } \\
\text { декаплинга, зеленый ВРП, } \\
\text { Истинные сбережения }\end{array}$ & 2010-2014 \\
\hline Korobitsyn, 2016 & Уральский ФО & Субъекты РФ & $\begin{array}{c}\text { Зеленый ВРП, Истинные } \\
\text { сбережения }\end{array}$ & 2010-2014 \\
\hline Korobitsyn, 2015 & Уральский ФО & Субъекты РФ & $\begin{array}{c}\text { Зеленый ВРП, Истинные } \\
\text { сбережения }\end{array}$ & 2010-2014 \\
\hline $\begin{array}{l}\text { Bobylev et } \\
\text { al., } 2012\end{array}$ & РФ в целом & Субъекты РФ & $\begin{array}{c}\text { Индекс скорректированных } \\
\text { чистых накоплений }\end{array}$ & Не указан \\
\hline $\begin{array}{l}\text { Ryumina, An- } \\
\text { ikina, } 2009\end{array}$ & РФ в целом & Субъекты РФ & $\begin{array}{c}\text { Экологически } \\
\text { скорректированные ВРП }\end{array}$ & 2003 \\
\hline Ryumina, 2020 & РФ в целом & Субъекты РФ & $\begin{array}{c}\text { Индекс человеческого } \\
\text { развития (ИЧР), } \\
\text { экологический ИЧР }\end{array}$ & 2016 \\
\hline Ryumina, 2018 & РФ в целом & Субъекты РФ & Экологический индекс & 2015 \\
\hline Alekseichuk, 2008 & Самарская область & Субъекты РФ & Чистые сбережения & $2000-2007$ \\
\hline
\end{tabular}


Продолжение таблицы 3

Continuation of table 3

\begin{tabular}{|c|c|c|c|c|}
\hline 1 & 2 & 3 & 4 & 5 \\
\hline Mekush, 2006 & $\begin{array}{c}\text { Кемеровская } \\
\text { область }\end{array}$ & Субъекты РФ & Истинные сбережения & 2001-2005 \\
\hline $\begin{array}{l}\text { Zabelina, 2020; } \\
\text { Zabelina, Delyuga, } \\
\text { 2019; Zabelina, } \\
\text { Kolotovkina, } 2019\end{array}$ & $\begin{array}{c}\text { Сибирский } \\
\text { и Дальневосточный } \\
\text { ФО }\end{array}$ & Субъекты РФ & $\begin{array}{c}\text { Уровень благополучия, } \\
\text { сводный экологический } \\
\text { индекс }\end{array}$ & 2017 \\
\hline Zander et al., 2010 & Красноярский край & Субъекты РФ & $\begin{array}{c}\text { Экологически } \\
\text { скорректированный ВРП }\end{array}$ & 2004-2006 \\
\hline $\begin{array}{l}\text { Syrtsova et } \\
\text { al., } 2018\end{array}$ & Сибирский ФО & Субъекты РФ & $\begin{array}{c}\text { Истинные сбережения, } \\
\text { истинный индикатор } \\
\text { прогресса }\end{array}$ & 2004-2013 \\
\hline $\begin{array}{l}\text { Syrtsova et } \\
\text { al., } 2016\end{array}$ & Сибирский ФО & Субъекты РФ & Истинные сбережения & 2013 \\
\hline $\begin{array}{l}\text { Belik, Priakh- } \\
\text { in, } 2013\end{array}$ & $\begin{array}{l}\text { Свердловская } \\
\text { область }\end{array}$ & Субъекты РФ & $\begin{array}{c}\text { Индекс скорректированных } \\
\text { чистых накоплений, } \\
\text { экологически } \\
\text { скорректированный ВРП }\end{array}$ & 2007-2012 \\
\hline $\begin{array}{l}\text { Galeeva et } \\
\text { al., } 2013\end{array}$ & $\begin{array}{c}\text { Республика } \\
\text { Татарстан }\end{array}$ & Субъекты РФ & Истинные сбережения & 2007-2011 \\
\hline $\begin{array}{l}\text { Khamatkha- } \\
\text { nov, } 2008\end{array}$ & $\begin{array}{l}\text { Республика } \\
\text { Ингушетия }\end{array}$ & Субъекты РФ & Истинные сбережения & 2000-2004 \\
\hline Barabash, 2013 & Рязанская область & Субъекты РФ & Коэффициент загрязнения & $2000-2010$ \\
\hline $\begin{array}{l}\text { Tkachev et } \\
\text { al., } 2018\end{array}$ & ХМАО-Югра & Субъекты РФ & Экологический след & $2010-2014$ \\
\hline
\end{tabular}

Преимущества и недостатки достаточно очевидны. В первом случае необходимо провести большую и не всегда в полной мере выполнимую работу по сопоставлению предложенных в других социальноэкономических реалиях показателей с действующей в стране системой статистического учета, но с перспективой на выходе получить оценки, которые можно будет использовать не только для анализа динамики устойчивости изучаемых объектов, но и для межстранового сопоставления. Второй подход лишен такого преимущества в обмен на высокую степень свободы при формировании способа расчета индикатора устойчивости. В то же время методические преимущества таких расчетов не очевидны, поскольку ни один из них не способен дать качественно новое представление о траектории устойчивости развития того или иного региона.
Первый подход реализован во многих вышеперечисленных работах. Например, широко используемые в международной литературе индексы «истинных сбережений», приведшие к созданию интегральных социо-эколого-экономических индексов «скорректированных чистых накоплений» (Adjusted Net Savings), рассчитаны для peгионов России коллективом С.Н. Бобылева на основе только официальной государственной статистической информации (Bobylev et al., 2012). Альтернативный подход к расчету «истинных сбережений» с учетом открытых данных об истощении природных ресурсов, предоставляемых компаниями-недропользователями, развит и успешно применен для регионов Сибири и Дальнего Востока на самом длинном доступном временном горизонте в работах (Pyzheva et al., 2021; Syrtsova et al., 2016). Экологически скорректированные оценки 
валовых региональных продуктов выполнены в циклах работ Е. В. Рюминой с соавт. (Ryumina, Anikina, 2009; Ryumina, 2018, 2020). В целом результаты указанных исследований совпадают в главном выводе: относительно богатые российские регионы демонстрируют существенные разрывы между уровнем своего экономического и социо-экологического благополучия. Рентные сверхдоходы, получаемые за счет торговли и использования на внутреннем рынке природных ресурсов, в самой малой доле инвестируются в создание комфортных условий для благополучия населения соответствующих территорий, включая экологическую составляющую. Сохранение такой диспропорции на горизонте многих лет приводит к накоплению нерешенных проблем, социальной и экологической деградации территорий. Применение оценок устойчивости позволяет дать количественное измерение таких диспропорций, их соотношений между разными территориями, на горизонте разных периодов времени.

Перечисленные работы ценны тем, что проводились для всей совокупности регионов страны, однако содержат пробелы в периодах соответствующих расчетов (часто не охватывают даже последнее десятилетие), что существенно ограничивает возможность использования данных peзультатов в целях формирования актуальной региональной политики. В то же время некоторые известные международные индексы, например Индекс «живой планеты» (Living Planet Index), Индикатор истинного прогресса (Genuine Progress Indicator, GPI), пока не нашли применения в России. В этом смысле особый интерес представляет последний индекс, который предназначен для измерения социо-эколого-экономического благополучия населения и был успешно применен во многих странах и регионах как альтернатива Валовому внутреннему продукту (Costanza et al., 2014). Методические расчет GPI представляется куда более сложной задачей, чем, например, оценка «истинных сбережений». Особенные трудности могут возникнуть при работе именно в региональном разрезе.
Результаты применения второго подхода, связанного с разработкой оригинальных российских индикаторов устойчивости, не представляют самостоятельного интеpeca, поскольку информации, имеющейся в соответствующих публикациях, недостаточно для того, чтобы сделать выводы о преимуществах соответствующих новых методик над теми, что являются адаптацией международных разработок. Как правило, суть работ, применяющих такой подход, сводится к сведению в один индекс наблюдающихся для региона статистических показателей, что далеко не всегда корректно с точки зрения следования концепции устойчивого развития.

Отдельно следует отметить, что большинство процитированных работ относятся к 2000-м, но не к 2010-м годам, что противоречит общей для российского и мирового академического сектора тенденции наращивания динамики публикаций, наблюдаемой в других научных областях. Причем нет не только обновленных оценок, но и попыток соответствующего обобщения результатов ранее выполненных работ. Также важно, что большинство упомянутых выше исследователей и коллективов, работы которых не получили логичного развития, продолжают заниматься активной научной деятельностью, но по другим тематикам.

Таким образом, можно констатировать, что наблюдается существенный дефицит исследований в области оценки устойчивости развития российских регионов, ликвидация которого представляется интересной перспективной задачей.

\section{Заключение}

Изложенные в статье результаты представляют, по всей видимости, первый комплексный обзор эмпирической литературы по анализу устойчивости развития российских регионов. Общие выводы проведенного исследования согласуются с результатами обзорно-библиометрических работ по другим секторам экономических наук в России, однако содержат и некоторые частные особенности. 
В целом российский рынок научной литературы в области экономики испытывает существенное влияние своей исторической изоляции от международного. Все авторы, занимавшиеся данной темой, едины во мнении, что за прошедшие с момента «открытия границ» три десятилетия так и не удалось преодолеть характерные проблемы, препятствующие опережающему развитию качественных научных академических исследований по экономике в России. Причины данного явления, разумеется, не специфичны именно для экономики, но выходят далеко за рамки нашей работы и связаны, прежде всего с тем, что реформы науки в стране и определенный прирост объемов ее совокупного финансирования не смогли переломить сложившиеся негативные практики и создать для отечественных ученых стимулы к занятию результативной и международноконкурентоспособной научной деятельностью. По-прежнему наблюдается сформировавшийся в 1990-е годы. острый дефицит молодых перспективных научных кадров, способных быстро адаптироваться к новым условиям: от необходимости свободного профессионального общения, чтения литературы и написания собственных материалов на английском языке до следования за тенденциями развития мирового исследовательского ландшафта. Кроме того, в России недостаточно качественных научных изданий, которые индексируются в ведущих международных базах данных, что также является важным сдерживающим фактором роста количества качественных научных публикаций.

В полной мере эта проблема проявляется для узкой тематики данного исследования. Несмотря на усиливающееся общественно-политическое внимание к проблемам устойчивости развития, экологизации экономики, повышения благополучия населения, интерес к данным вопросам в российском академическом секторе растет не настолько очевидными темпами, а по некоторым показателям - стагнирует. Результаты исследования наглядно демонстрируют, что проблемы устойчивости развития регионов достаточно периферийны и не являются мейнстримом российской экономической науки. Большая часть исследований носит постановочный или описательный характер, а количественных исследований, столь востребованных на современном международном научном рынке, очень мало. Например, оценками уровней устойчивости развития покрыта лишь малая часть российских регионов, причем за очень небольшой период, не превышающий в среднем пяти лет и с применением очень узкого круга инструментов. Этого явно недостаточно для использования в качестве альтернатив традиционным макроэкономическим мерам благополучия развития территорий, что существенно сужает востребованность данных результатов практикой.

Любопытно, что исследованиями по данной тематике не занимаются даже зарубежные исследователи, интерес которых сосредоточен на приграничных странах, как это часто бывает в других областях экономической науки. Наши бывшие соотечественники, работающие теперь в зарубежных вузах и научных институтах, не спешат заниматься данной тематикой по России, а зарубежные исследователи и вовсе не проявляют к ней интереса, фокусируясь на проблемах своих стран или глобальной повестки. Такая ситуация закономерна: как правило, исследованиями территориально-специфических вопросов занимаются представители местных научных коллективов, но с привлечением в том числе коллег из других стран. Следовательно, в рамках такой логики дефицит публикаций по тематике устойчивости российских регионов можно воспринимать как направление для развития соответствующих исследований, открытия новых направлений и даже создания новых коллективов. 


\section{Список литературы / References}

Adam, A.M., Laptev, N.I. (2015). Otsenka ustoichivosti razvitiia Tomskoi oblasti po indeksu ustoichivosti [Assessment of the sustainability of Tomsk region's development according to the sustainability index]. In Teoreticheskie i prikladnye aspekty sovremennoi nauki [Theoretical and applied aspects of modern science], 7-2, 122-124.

Alekseichuk, M.S. (2008). Chistye sberezheniia kak indikator otsenki ustoichivosti regionalnogo razvitiia [Net Savings as an Indicator for Assessing the Sustainability of Regional Development]. In Region: ekonomika i sotsiologiia [Region: Economics and Sociology], 3, 67-77.

Ali, F., Ashfaq, M., Begum, S., Ali, A. (2020). How «Green» thinking and altruism translate into purchasing intentions for electronics products: The intrinsic-extrinsic motivation mechanism. In Sustainable Production and Consumption, 24, 281-291, DOI: 10.1016/j.spc.2020.07.013.

Aria, M., Cuccurullo, C. (2017). Bibliometrix: An R-tool for comprehensive science mapping analysis. In Journal of Informetrics, 11(4), 959-975. DOI: 10.1016/j.joi.2017.08.007

Barabash, D.A. (2013). Kompleksnaia otsenka ekonomicheskoi effektivnosti i opredelenie ustoichivosti razvitiia regionalnoi ekonomiki [Comprehensive assessment of economic efficiency and determination of the sustainability of regional economic development]. In Vestnik Finansovogo universiteta [Bulletin of the Financial University], 5 (77), 149-154.

Belik, I.S., Priakhin, D.A. (2013). Sotsialno-ekologicheskaia sostavliaiushchaia ustoichivogo razvitiia regiona [Socio-environmental component of the region's sustainable development]. In Ekonomika regiona [Economy of Region], 3, 142-152. DOI: 10.17059/2013-3-12.

Bobylev, S.N., Kudriavtseva, O.V., Soloveva, S.V. (2014). Indikatory ustoichivogo razvitiia dlia gorodov [Sustainable Development Indicators for Cities]. In Ekonomika regiona [Economy of Region], 3, 101110. DOI: 10.17059/2014-3-9.

Bobylev, S.N., Kudryavtseva, O.V., Solovyeva, S.V., Sitkina, K.S. (2018). Indikatory ekologicheski ustoichivogo razvitiia: regional'noe izmerenie [Sustainable development indicators: regional dimension]. In Vestnik Moskovskogo universiteta. Seriia 6: Ekonomika [Herald of the Moscow State University. Series 6. Economics], 2, 21-33.

Bobylev, S.N., Minakov, V.S., Solovyeva, S.V., Tretyakov, V.V. (2012). Ekologo-ekonomicheskii indeks dlia regionov Rossii [Ecological and economic index of Russian regions. Calculation methodology and indicators]. Moscow, WWF Russia, RIA Novosti.

Bobylev, S.N., Soloveva, S.V., Sitkina, K.S. (2013). Indikatory ustoichivogo razvitiia Uralskogo regiona [Indicators of sustainable development of the Urals region]. In Ekonomika regiona [Economy of Region], 2, 10-17. DOI: $10.17059 / 2013-2-1$.

Carayannis, E.G., Cherepovitsyn, A.E., Ilinova, A.A. (2017). Sustainable Development of the Russian Arctic zone energy shelf: the Role of the Quintuple Innovation Helix Model In Journal of the Knowledge Economy, 8 (2), 456-470. DOI: 10.1007/s13132-017-0478-9.

Costanza, R., Kubiszewski, I., Giovannini, E., Lovins, H., McGlade, J., Pickett, K. E., Ragnarsdóttir, K. V., Roberts, D., De Vogli, R., Wilkinson, R. (2014). Time to leave GDP behind. In Nature, 505(7483), 283-285. DOI: $10.1038 / 505283$ a.

Demyanenko, A.N., Demyanenko, N.A., Ukrainskiy, V.N. (2012). Rossiiskaia prostranstvennaia ekonomika: bibliometricheskii analiz [Russian spatial economy: bibliometric analysis]. In Prostranstvennaia ekonomika [Spatial economy], 3, 111-134.

Dubina, I.N., Campbell, D.F.J., Carayannis, E.G., Chub, A.A., Grigoroudis, E., Kozhevina, O.V. (2017). The Balanced Development of the Spatial Innovation and Entrepreneurial Ecosystem Based on Principles of the Systems Compromise: A Conceptual Framework. In Journal of the Knowledge Economy, 8(2), 438-455.

Eker, S. et al. (2019). Model validation: A bibliometric analysis of the literature. In Environmental Modelling \& Software,117, 43-54. DOI: 10.1016/j.envsoft.2019.03.009.

Gafurov, I.R., Safiullin, M.R., Elshin, L.A., Prygunova, M.I., Egorov, D.O. (2015). Effektivnost sotsialno-ekonomicheskogo razvitiia regionov Privolzhskogo federalnogo okruga: ustoichivost i tendentsii 
razvitiia [Efficiency of Socio-Economic Development of the Regions of the Volga Federal District: Sustainability and Development Trends]. Kazan, 112 p.

Galeeva, A.R., Gazizova, O.V. (2013). Istinnye sberezheniia kak indikator ustoichivogo razvitiia regiona [Genuine savings as an indicator of sustainable development of the region]. In Vestnik Kazanskogo tekhnologicheskogo universiteta [Bulletin of Kazan Technological University], 16(23), 258-261.

Glazyrina, I.P. et al. (2005). Ekologicheskie indikatory kachestva rosta regional'noj ekonomiki [Quality of growth indicators for regional economies]. Moscow, NIA-Priroda, $192 \mathrm{p}$.

Grigor'eva E.I., Glukhov V. A. (2017). RINZ menyaetsya [The RSCI is changing]. In Vlast' (The authority), 25(5), 221-223.

Hák, T., Janoušková, S., Moldan, B., Dahl, A.L. (2018). Closing the sustainability gap: 30 years after «Our Common Future», society lacks meaningful stories and relevant indicators to make the right decisions and build public support. In Ecological Indicators, 87, 193-195. DOI: 10.1016/j.ecolind.2017.12.017.

Hallinger, P.A. (2021). Meta-Synthesis of Bibliometric Reviews of Research on Managing for Sustainability, 1982-2019. In Sustainability, 13, 3469. DOI: 10.3390/su13063469

Hecht, A.D. (1999). The Triad of Sustainable Development: Promoting Sustainable Development in Developing Countries. In Journal of Environment \& Development, 8(2), 111-132. DOI: $10.1177 / 107049659900800202$.

Khamatkhanov, T.M. (2008). Otsenka ekologo-ekonomicheskoi ustoichivosti Respubliki Ingushetiia na osnove primeneniia pokazatelia istinnykh sberezhenii [Assessment of the environmental and economic sustainability of the Republic of Ingushetia based on the application of the indicator of true savings]. In Vestnik Cheliabinskogo gosudarstvennogo universiteta [Bulletin of Chelyabinsk State University], 29, 69-77.

Kolomak, E.A. (2019). Prostranstvennoe razvitie Rossii v XXI v [Spatial Development of Russia in the XXI]. In Prostranstvennaia ekonomika [Spatial Economics], 15(4), 85-106. DOI: 10.14530/se.2019.4.085106.

Korobitsyn, B.A. (2015). Metodicheskii podkhod k uchetu istoshcheniia prirodnykh resursov, izmeneniia sostoianiia okruzhaiushchei sredy i chelovecheskogo kapitala $\mathrm{v}$ valovom regionalnom produkte [Methodological approach to accounting for the depletion of natural resources, environmental change, and human capital in the gross regional product]. In Ekonomika regiona [Economy of Region], 3, 77-88. DOI: $10.17059 / 2015-3-7$.

Korobitsyn, B.A. (2016). Korrektirovka makroekonomicheskikh pokazatelei Rossiiskoi federatsii s uchetom istoshcheniia prirodnykh resursov, negativnogo vozdeistviia na okruzhaiushchuiu sredu i izmeneniia chelovecheskogo kapitala [Adjustment of macroeconomic indicators of the Russian Federation taking into account the depletion of natural resources, the negative impact on the environment and changes in human capital]. In Ekonomika prirodopolzovaniia [Economics of natural resource use], 5, 63-76.

Korobitsyn, B.A. (2018). «Zelenaia» ekonomika, indikatory «zelenogo» rosta i ikh dinamika v Uralskom federalnom okruge [«Green» economy, «green» growth indicators and their dynamics in the Ural Federal District]. In Biosfernaia sovmestimost: chelovek, region, tekhnologii [Biosphere compatibility: man, region, technology], 1(21), 107-114.

Kovalenko, E.G. et al. (2016). Problems and Mechanisms of Sustainable Development of Rural Areas (at the example of the Republic of Mordovia). In European Research Studies Journal, XIX(3A), 110-122.

Kryukov, V.A., Kolomak, E.A. (2021). Prostranstvennoe razvitie Rossii: osnovnye problemy i podkhody k ikh preodoleniiu [Spatial development of Russia: main problems and approaches to overcoming them]. In Nauchnye trudy volnogo ekonomicheskogo obshchestva Rossii [Scientific Proceedings of the Free Economic Society of Russia], 227(1), 92-114. DOI: 10.38197/2072-2060-2021-227-1-92-114.

Maksimov Yu., Mityakov S., Mityakov Ye. (2011). The system of indicators of sustainable development in the region. In Economy of region, 1(2), 226-231. DOI: 10.17059/2011-2-28.

Malik, A.S. (2012). Sustainable Development: Ecology and Economic Growth. Handbook of Climate Change Mitigation, 197-233. DOI: 10.1007/978-1-4419-7991-9_7.

Mekush, G.E. (2006). Podkhody k razrabotke indikatorov ustoichivogo razvitiia na regionalnom urovne (na primere Kemerovskoi oblasti) [Approaches to the development of indicators of sustainable de- 
velopment at the regional level (on the example of the Kemerovo region)]. In Geografiia i prirodnye resursy [Geography and natural resources], 1, 18-24.

Mkrtchyan, G.M., Lychagin, M.V., Lychagin, A.M. (2015). Novoe v issledovanii ekonomiki okruzhaiushchei sredy v 2006-2013 godakh: bibliometricheskii analiz na osnove Ecolit [New in Environmental Economics Study 2006-2013: bibliometric analysis based on Econlit]. In Mir ekonomiki i upravleniia [World of Economics and Management], 15(1), 131-143.

Muravyev, A.A. (2011). O rossiiskoi ekonomicheskoi nauke skvoz prizmu publikatsii rossiiskikh uchenykh v otechestvennykh i zarubezhnykh zhurnalakh za 2000-2009 gg. [On Russian economic science through the prism of publications of Russian scientists in Russian and foreign journals for 2000-2009]. In Ekonomicheskiy zhurnal VShE [Economic Journal HSE], 2, 237-264.

Pyzhev, A.I. (2021). Studies on the Russian forest industry: Bibliometric analysis. In Terra Economicus, 19(1), 63-77. DOI: 10.18522/2073-6606-2021-19-1-63-77.

Pyzheva, Yu.I., Lapo, E.V., Syrtsova, E.A., Pyzhev, A.I. (2021). Evaluation of Genuine Savings in the Russia's Far East Regions. In Regional Research of Russia, 11(1), 121-128. DOI: 10.1134/S2079970521010111

Quental, N., Lourenço, J.M. (2012). References, authors, journals and scientific disciplines underlying the sustainable development literature: a citation analysis. In Scientometrics, 90, 361-381. DOI: 10.1007/ s11192-011-0533-4.

R Core Team (2020). R: A language and environment for statistical computing. R Foundation for Statistical Computing, Vienna, Austria. Available at: https://www.R-project.org.

Ryumina, E.V. (2018). Ekologicheskii indeks: postroenie i ispolzovanie pri analize kachestva zhizni $i$ kachestva naseleniia [Ecological index: construction and use in the analysis of the quality of life and quality of population]. In Upravlenie ekonomicheskimi sistemami: elektronnyi nauchnyi zhurnal [Management of economic systems: electronic scientific journal], 9(115).

Ryumina, E.V. (2020). Ekologicheski skorrektirovannyi indeks chelovecheskogo razvitiia [Ecologically Adjusted Human Development Index]. In Narodonaselenie [Population], 23(1), 4-12. DOI: 10.19181/ population.2020.23.1.1.

Ryumina, E.V., Anikina, A.M. (2009). Ekologicheski skorrektirovannaya otsenka eknomicheskogo razvitiya regionov [Ecologically adjusted assessment of the economic development of the regions]. In Problemy prognozirovaniia [Problems of forecasting], 2(113), 78-94.

Shadrina, E. (2015). Russia's pivot to Asia: Rationale, progress, and prospects for oil and gas cooperation. In Region: Regional Studies of Russia, Eastern Europe, and Central Asia, 4(1), 95-128.

Syrtsova, E.A., Pyzhev, A.I., Zander, E.V. (2016). Istinnye sberezheniia regionov Sibiri: novye otsenki, starye problemy [Genuine savings for Siberian regions: new estimates, old problems]. In EKO [ECO], 6(504), 109-129.

Syrtsova, E.A., Pyzhev, A.I., Zander, E.V., Pyzheva, Iu.I. (2018). Kompleksnaia otsenka ustoichivosti razvitiia sotsio-ekologo-ekonomicheskikh sistem regionov Sibiri [Comprehensive Assessment of the Sustainability of Socio-Economic Systems of Siberia's Regions], Krasnoyarsk.

Tambovceva, T., Dimante, D., Atstāja, D. (2018). Consumer behaviour change through education for sustainable development: Case of Latvia. In International Journal of Environmental Technology and Management, 21(5-6), 238-252.

Tkachev, B.P., Zaitseva, A.V., Tkacheva, T.V. (2018). Raschet ekologicheskogo sleda v KhantyMansiiskom okruge-Iugre [Calculation of the ecological footprint in the Khanty-Mansi Autonomous Okrug-Yugra]. In Uspekhi sovremennogo estestvoznaniia [Advances of modern natural science], 11-2, 395-399.

UNDP's Strategy for Inclusive and Sustainable Growth. United Nations Development Programme. 2017. Available at: https://www.undp.org/publications/undps-strategy-inclusive-and-sustainable-growth

Uskova, T.V. (2009). Upravlenie ustoichivym razvitiem regiona [Managing the sustainable development of the region]. Vologda.

Wang, Y., Chen, Yu., Meijun, L., Hu, R. (2018). Growth and quality of Chinese journals from 1949 to 2013. In Learned Publishing, 31(3), 205-214. DOI: 10.1002/leap.1162. 
Yakovenko, N.V., Ten, R.V., Komov, I.V., Didenko, O.V. (2021). Ustoichivost sotsialno-ekonomicheskogo razvitiia munitsipalnykh obrazovanii Voronezhskoi oblasti [Sustainability of socio-economic development of municipalities of the Voronezh region]. In Iug Rossii: ekologiia, razvitie [South of Russia: ecology, development], 16(1), 87-97. DOI:10.18470/1992-1098-2021-1-87-97.

Zabelina, I.A. (2020). Ispolzovaniia gis-instrumentariia $v$ issledovanii sotsio-ekologo ekonomicheskogo blagopoluchiia regionov Sibiri i Dalnego Vostoka [The Use of GIS Toolkit in the Study of SocioEconomic Well-Being of Siberia and the Far East Regions], In Ekologiia. Ekonomika. Informatika. Seriia: Geoinformatsionnye tekhnologii i kosmicheskii monitoring [Ecology. Economics. Informatics. Series: Geoinformation technologies and space monitoring], 2(5), 14-20. DOI: 10.23885/2500-123X-2020-2-5$14-20$.

Zabelina, I.A., Deluga, A.V. (2019). Geoekologicheskie indikatory ustoichivogo razvitiia: prostranstvennyi analiz [Geoecological indicators of sustainable development: spatial analysis]. In Ustojchivoe razvitie gornyh territorij [Sustainable development of mountain territories], 11(1), 15-25. DOI: 10.21177/19984502-2019-11-1-15-25.

Zabelina, I.A., Kolotovkina, Yu.V. (2019) Ekologo-ekonomicheskoe razvitie munitsipal'nykh obrazovanii na syr'evykh territoriiakh Zabaikal'skogo kraia v kontekste kontseptsii «zelenoi» ekonomiki [Ecological and economic development of municipalities of the Zabaykalsky krai in the context of «green» economy]. In Izvestiia Ural'skogo gosudarstvennogo gornogo universiteta [Izvestiya Ural'skogo gosudarstvennogo gornogo universiteta], 1(53), 149-155. DOI 10.21440/2307-2091-2019-1-149-155.

Zander E. V., Startseva Yu.I., Pyzhev A. I. (2010). Green GRP as a Macroeconomic Indicator of Economic Growth of a Region (by the Example of Krasnoyarsk krai). In Journal of Siberian Federal University. Humanities \& Social Sciences, 3, 382-387. 